\title{
Resonance Effect in Isotropic Phase of Lyotropic Liquid Crystal
}

\author{
D.A. de Oliveira ${ }^{1}$ and P.R.G. Fernandes ${ }^{2}$ \\ ${ }^{1}$ CEFET, Campo Mourão, Paraná, Brazil \\ ${ }^{2}$ Departamento de Física, Universidade Estadual de Maringá \\ Av. Colombo, 5790, Maringá, Paraná, Brazil, 87020-900
}

Received on 15 November, 2001

\begin{abstract}
In this work we present experimental results that show a resonance effect in the isotropic phase of a lyotropic liquid crystal. The lyotropic mixture is made up of Potassium Laurate (KL), Decanol $(\mathrm{DeOH})$ and water with three different relative concentrations: $2.62,2.79$ and $2.90(\mathrm{C}=$ $[\mathrm{KL}] /[\mathrm{DeOH}],[\mathrm{KL}]$ and $[\mathrm{DeOH}]$ in $\%$ molar). These mixtures have an isotropic (ISO) phase between two lamellar phases as a function of the temperature. From the transmittance of the sample $(I)$ as a function of the frequency $(f)$ it was possible to evaluate a characteristic time $(\tau)$ in order of magnitude as a function of the temperature $(T)$. The existence of a maximum in $\tau$ vs. $T$ around $30^{\circ} \mathrm{C}$ indicates the possibility of employing lyotropic liquid crystals in mechanical vibration sensors. We are proposing a mechanical vibration sensor able to measure low mechanical frequencies $(f \leq 200 \mathrm{~Hz})$.
\end{abstract}

\section{Introduction}

Liquid crystals have a complex flow regime because the translational motions are coupled to the orientational motions of their basic structures [1]. The combination of fluid mobility with the anisotropy of physical properties has made them an interesting object for scientific research and technical applications. Nematic liquid crystal has a large range of applications, from displays (LCDs) to pressure and temperature sensors [2]. In particular, liquid crystal sensors for pressure measurements have recently been made using a polarization effect caused by the strong rotatory power of chiral nematic liquid crystal [3] and the light polarization modulation effect in nematic liquid crystal [4]. From a technological point of view lyotropic liquid crystals do not have as many applications as thermotropic [5] or polymeric liquid crystals [6]. By consulting patent data bases we have found few technological applications using lyotropics. Among them there is one using a quaternary mixture to obtain thermally, electrically or magnetically controllable optical devices [7]. Some efforts have been made to understand the behavior of complex fluids under flow [8,9] and micellar complex fluids are only beginning to be investigated [10-12].

Lyotropic liquid crystals (LLCs) are very interesting, showing as they do rich phase diagrams [13] as a function of temperature and of the relative concentration of each compound in the mixture. The nematic $(\mathrm{N})$ regions of the LLC phase diagrams are usu- ally surrounded by isotropic domains. The isotropic phase (ISO) of our lyotropic mixture of potassium laurate $(\mathrm{KL})$, decanol $(\mathrm{DeOH})$ and water is found between two lamellar phases. In the ISO phase of this mixture it is possible to induce birefringence [14] by flow, and this effect is also observed in optically isotropic lyotropic sponge phases [15] and thermotropic liquid crystals [16]. Recently, we have presented experimental results and a theoretical study [17] of the flow-induced birefringence in the ISO phase of a lyotropic liquid mixture to explain the experimental results obtained in reference [14]. Once the shear flow has vanished, the magnitude of the director relaxes and the sample comes back to the ISO phase with a typical relaxation time $\tau \sim 10^{-2} \mathrm{~s}$. From a theoretical point of view this problem was treated in reference [17] as a diffusion of the tensor order parameter inside the sample, and a characteristic length, $l \sim 10^{-4} \mathrm{~cm}$, was obtained. Using Langevin's equation, Simões et al [18] showed that the induced order can be understood as a result of two distinct competing causes: the coherent torques caused by the external fields and the Brownian aleatory fluctuations that look for the destruction of any uniformity.

In this work we present an experimental study of induced birefringence by mechanical stresses in the isotropic phase of lyotropic liquid crystals. Using a bandwidth approach it was possible to determine a characteristic time $(\tau)$ that is associate to the response of the micelles to the driving force with a specific frequency. We explore this effect to produce a mechanical 
vibration liquid crystal sensor at room temperature $(\sim$ $25^{\circ} \mathrm{C}$ ) [20] using LLCs. This sensor can measure low mechanical frequencies $(f \leq 200 \mathrm{~Hz})$ and paves the way for the future application of lyotropic liquid crystals in low frequency controllable technological devices.

\section{Experimental}

The lyotropic liquid crystals (M1, M2 and M3) used in this work are mixtures of potassium laurate (KL), decanol(DeOH) and water. The relative concentrations $\mathrm{C}$ $=[\mathrm{KL}] /[\mathrm{DeOH}]$, where $[\mathrm{KL}]$ and $[\mathrm{DeOH}]$ are given in $\%$ molar, of each mixture are show in Table 1.

Table 1 - Relative concentrations $\mathrm{C}=[\mathrm{KL}] /[\mathrm{DeOH}]$ ([KL] and $[\mathrm{DeOH}]$ in \% molar) of each mixture

\begin{tabular}{|c|c|}
\hline Mixture & $\mathrm{C}=[\mathrm{KL}] /[\mathrm{DeOH}]$ \\
\hline \hline M1 & 2.62 \\
\hline M2 & 2.79 \\
\hline M3 & 2.90 \\
\hline
\end{tabular}

The phase sequences as a function of temperature (T), determined by optical and X-ray scattering techniques, are given in Table 2, where ISO is isotropic phase, $\mathrm{L}_{1}$ and $\mathrm{L}_{2}$ are lamellar phases at low and high temperatures respectively.

Table 2 - Phase sequence as a function of temperature of each mixture; $\mathrm{L}_{1}$ and $\mathrm{L}_{2}$ are lamellar phases; ISO is isotropic phase.

\begin{tabular}{|c|c|c|c|c|c|}
\hline Mixture & & $\mathrm{T}_{C}\left({ }^{\circ} \mathrm{C}\right)$ & & $\mathrm{T}_{C}\left({ }^{\circ} \mathrm{C}\right)$ & \\
\hline \hline M1 & $\mathrm{L}_{1}$ & 9.5 & ISO & 54.0 & $\mathrm{~L}_{2}$ \\
\hline M2 & $\mathrm{L}_{1}$ & 8.5 & ISO & 49.0 & $\mathrm{~L}_{2}$ \\
\hline M3 & $\mathrm{L}_{1}$ & 9.5 & ISO & 52.0 & $\mathrm{~L}_{2}$ \\
\hline
\end{tabular}

The set up to measure the transmittance of the sample as a function of frequency when it undergoes an external mechanical vibration is sketched in Fig. 1. The lyotropic mixture $(\mathrm{V}=1 \mathrm{ml})$ is encapsulated in a sample holder glass(Hellma) with a rectangular section shape. The cell is carefully sealed to avoid concentration gradients in the sample due to losses of water or decanol. A polarized light beam (He-Ne laser, $\lambda=632.8 \mathrm{~nm}$ ) illuminates the sample in the $\mathrm{x}$ direction. A function generator (Stanford DS335) is coupled to a loud-speaker and to an iron wire. The wire touches the cell and promotes the movement of the sample. The external oscillations are always perpendicular to the laser beam direction. The oscillations are parallel to the z-axis of the laboratory frame (see Fig. 1). Beyond the analyzer, a photodiode detects the light intensity that comes mainly from the induced birefringence, and a lock-in amplifier (Stanford SR830) is used to analyse the signal. The cell is placed on a temperature controlled device $\left( \pm 0.2^{0} \mathrm{C}\right.$ stability). The external force applied to the cell is a square wave of $5.0 \mathrm{~V}_{r m s}$ with frequencies between $0 \mathrm{~Hz}$ to $200 \mathrm{~Hz}$. The whole set up is placed on an optical table isolated from spurious vibrations.

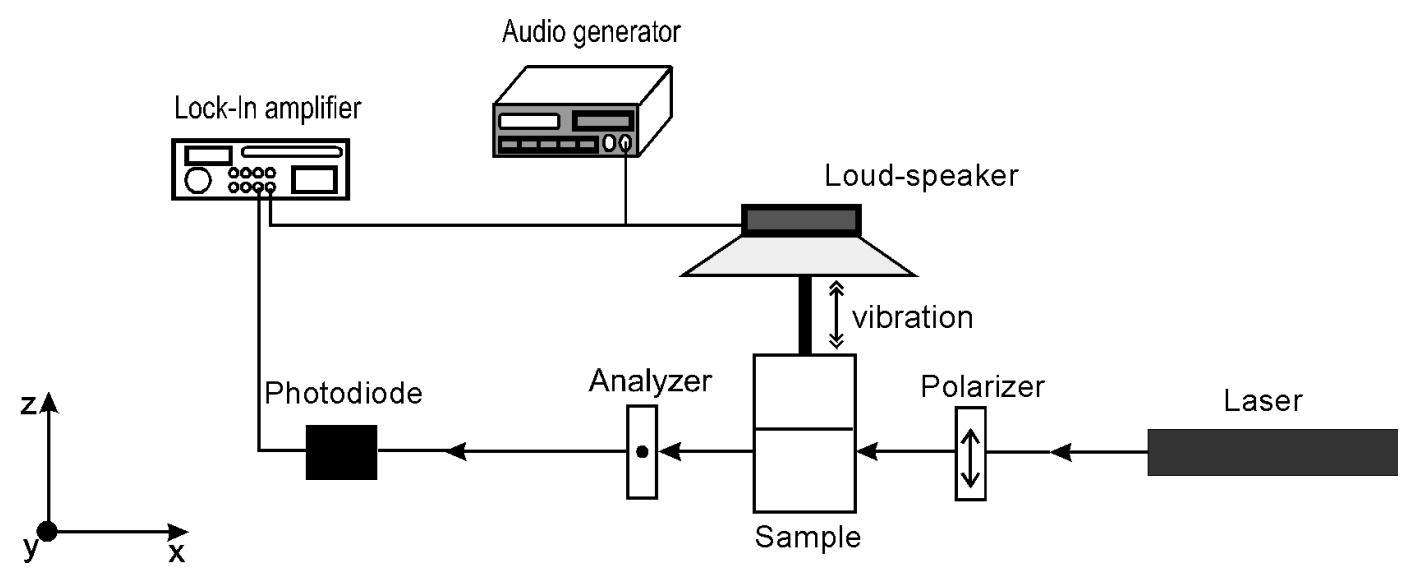

Figure 1. Experimental set up to measure the sample transmittance as a function of time.

The methodology of the experiment is as follows: taking a sample (from Table 1) and fixing the temperature and frequency we introduce the oscillations in the z-direction (vertical axis); simultaneously the laser beam passes through the sample in the $\mathrm{x}$-direction. The direction of the inciding polarization is parallel to the z-axis. A photodiode connected to a lock-in amplifier detects the transmitted light as a function of frequency and temperature. 


\section{Results and discussions}

Figure 2 shows plots of transmittance $(I)$ as a function of frequency $(f)$ with M1, M2 and M3 mixtures at $20^{\circ} \mathrm{C}$. By changing the temperature we have seen that $I$ increase in the middle of the ISO phase $\left(30^{\circ} \mathrm{C}\right)$ and decrease near the lamellar phases. The present experimental study concentrated on the low frequency range $(f \leq 200 \mathrm{~Hz})$ but more vibrational modes were observed between $0 \mathrm{~Hz}$ and $1 \mathrm{kHz}$. The errors were evaluated taking into account the reproducibility of the experiments. The experimental error was $0.3 \%$ in each bit of data in Fig. 2. In addition, some fluctuations in the maximum value of $I$ where observed. These fluctuations were about $10 \%$ around $30^{\circ} \mathrm{C}$ and about $1.0 \%$ near the lamellar phases. Such fluctuations in $I$ may be reflecting the instability of the induced order in the ISO phase. Fig. 2 shows sharp peaks in some specific frequencies. We identify such frequencies (resonance frequencies) as possible ones to induce order in the ISO phase of the mixtures in Table 1 . The values of the resonance frequencies, $f$ and its deviation, $\Delta f$ have been found as: $(20 \pm 2) \mathrm{Hz},(120 \pm 1) \mathrm{Hz}$ and $(180 \pm 1) \mathrm{Hz}$. These frequencies presented the same values when the temperature or relative concentration of the mixture was changed. In addition, Fig. 2 shows a broad peak around $60 \mathrm{~Hz}$, which was not investigated in this work.

In order to evaluate the magnitude of the characteristic time $(\tau)$ of induced birefringence by mechanical stresses in the ISO phase we take the resonance approach [19] where the bandwidth of the maximum transmittance peak of Fig. 2 represents the response of the micelles to the driving force, considering the low viscosity of the system [17]. This way, taking the inverse of bandwidth of the transmittance spectrum we evaluated $\tau$ in order of magnitude. Fig. 3 shows $\tau$ as a function of temperature $(T)$ using the mixture M3 and $120 \mathrm{~Hz}$. The mixture M3 showed the best experimental results where $\tau$ is seconds in order of magnitude. The error in $\tau$ showed in Fig. 3 was $5 \%$. By changing the temperature we can see an increase in $\tau$ near the lamellar phases and around $30^{\circ} \mathrm{C}$ (middle of the ISO phase). The increase in $\tau$ near to lamellar phases and the peak at $30^{\circ} \mathrm{C}$ indicates that isotropic phase has different correlation properties. We have been observed that the graph of the characteristic time as a function of temperature presents the same topology when we compare $\tau$ vs. $T$ using a steady-state with another one using a transient regime [14] with the same mixture. This topological similarity indicates that the increase of $\tau$ near to ordered phases independs of the frequencies. The increase in $\tau$ near $30^{\circ} \mathrm{C}$ confirms the possibility of employing lyotropic liquid crystals in technological applications as a mechanical vibration sensors. We have developed a device to detect low mechanical vibrations $(f \leq 200 \mathrm{~Hz})[20]$ using the mixtures of the Table 1. The stability of the lyotropic mixtures has been the main problem to our mechanical vibration sensor. To overcome these problems other mixtures are being investigated. The mechanical vibration sensor we have developed paves the way for the use of lyotropic liquid crystals in low frequency controllable technological devices.

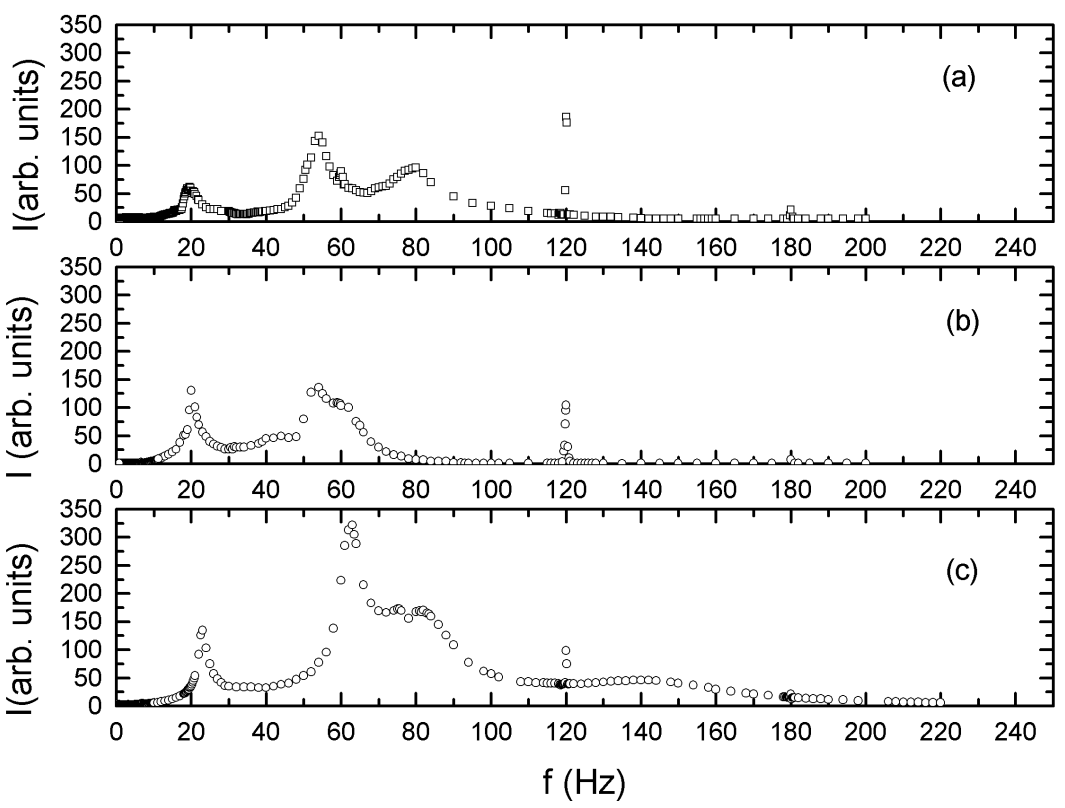

Figure 2. Transmittance $(I)$ in arbitrary units as a function of frequency for three relative concentrations, $\mathrm{C}=[\mathrm{KL}] /[\mathrm{DeOH}]$ : (a) 2.90 ; (b) $2.79 ;$ (c) $2.62 ; T=20^{\circ} \mathrm{C}$. 


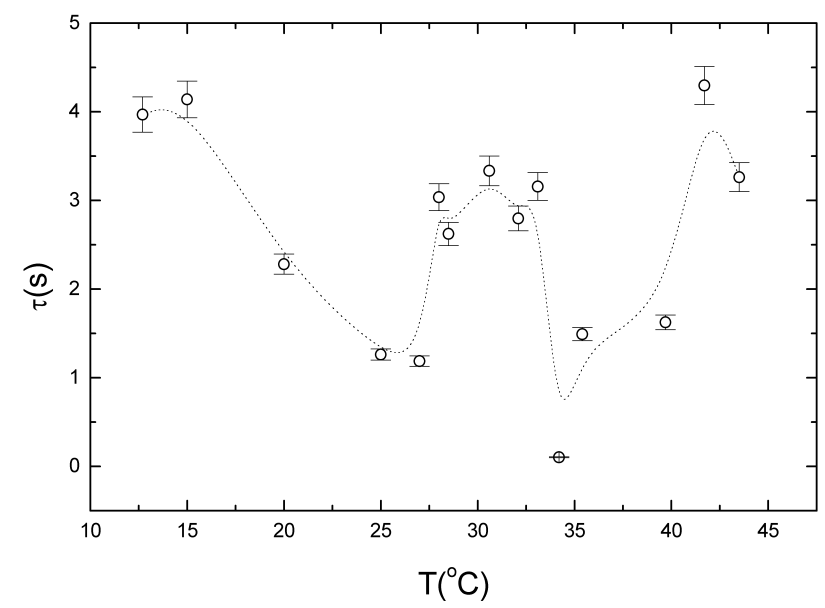

Figure 3. Characteristic time as a function of temperature; $\mathrm{C}=2.90 ; f=(120 \pm 1) \mathrm{Hz}$; The dot line is only a guide of eyes.

\section{Conclusion}

A resonance effect was experimentally verified in the isotropic phase of $\mathrm{KL} / \mathrm{DeOH} /$ water mixture in three different concentrations. This effect independs of the relative concentration of mixtures and of temperature. Using a bandwidth approach it was possible to evaluate the characteristic time $(\tau)$ in order of magnitude that was associate to the response of the micelles to the driving force. The existence of a maximum in the $\tau$ vs. $T$ around $30^{\circ} \mathrm{C}$ indicates the possibility of employing lyotropic liquid crystals in mechanical vibration sensors. As far as we know we are proposing the first application for lyotropic liquid crystal to measure mechanical vibrations.

\section{Acknowledgments}

We thank PADCT and CNPq for financial support.

\section{References}

[1] P.G. de Gennes and A.J. Prost, The Physics of Liquid Crystal, (Clarendon, Oxford, 2nd. Ed. 1993).
[2] Cerwin, Stephen A., USPatent, Appl. No. 911216, November, 16 (1999).

[3] T.R. Wolinski, A. Jarmolik, and W. J. Bock, IEEE Transactions on Instrumentation and Measurements, 48, 1 (1999).

[4] O.A. Kapustina et al., Mol. Cryst. Liq. Cryst. 209, 19 (1991).

[5] M. Kawamura and S. Sato, Jpn. J. Appl. Phys. 37, 5669 (1998); Y.V. Bocharov et al., Sensors and Actuators A, 28, 179 (1991).

[6] R.A.M. Hikmet and H. Kemperman, Nature 392, 476 (1998).

[7] James Tabony, USPatent, Appl. No. 113, 288, November, 8 (1988).

[8] A.D. Rey and M.M. Denn, Mol. Cryst. Liq. Cryst. 153, 301 (1987); Liq. Cryst. 4, 53 (1989).

[9] P.D. Olmsted and C. -Y. D. Lu, Phys. Rev. E 56, R55 (1997).

[10] S.Q. Wang, J. Phys. Chem. 94, 8382 (1990).

[11] K.A. Coppi, M. Tirrel, and F.S. Bates, Phys. Rev. Lett. 70, 1449 (1993).

[12] E. Peuvrel and P. Navard, Liquid Crystal 7, 95 (1990).

[13] L.J. Yu and Saupe, Phys. Rev. Lett. 45, 1000 (1980).

[14] P.R.G. Fernandes and A.M. Figueiredo Neto, Phys. Rev. E 51, 567 (1995).

[15] G. Porte, J. Appell, P. Bassereau, and J. Marignan, J. Phys. France 50, 1335 (1989).

[16] P. Martinoty and M. Bader, J. Phys. (Paris) Colloq. 42, 1097 (1981).

[17] P.R.G. Fernandes and A.M. Figueiredo Neto, Phys. Rev. E 56, 6185 (1997).

[18] M. Simões, P.R.Fernandes, A.J. Palangana, and S.M. Domiciano, Phys. Rev. E 64, 021707 (2001).

[19] I. G. Main, Vibrations and waves in physics, Cambridge University Press (1978)

[20] P.R.G. Fernandes, Brazilian Patent, PI 9805500-3, November, 20 (1998) 